\title{
Evaluating the outcomes of a podiatry-led assessment service in a public hospital orthopaedic unit
}

\author{
Daniel R Bonanno ${ }^{1,2,3^{*}}$, Virginia G Medica ${ }^{3}$, Daphne S Tan ${ }^{1}$, Anita A Spring ${ }^{3}$, Adam R Bird ${ }^{1,2}$ and Jana Gazarek ${ }^{3}$
}

\begin{abstract}
Background: In Australia, the demand for foot and ankle orthopaedic services in public health settings currently outweighs capacity. Introducing experienced allied health professionals into orthopaedic units to initiate the triage, assessment and management of patients has been proposed to help meet demand. The aim of this study was to evaluate the effect of introducing a podiatry-led assessment service in a public hospital orthopaedic unit. The outcomes of interest were determining: the proportion of patients discharged without requiring an orthopaedic appointment, agreement in diagnosis between the patient referral and the assessing podiatrist, the proportion of foot and ankle conditions presenting to the service, and the proportion of each condition to require an orthopaedic appointment.
\end{abstract}

Methods: This study audited the first 100 patients to receive an appointment at a new podiatry-led assessment service. The podiatrist triaged 'Category 3 ' referrals consisting of musculoskeletal foot and ankle conditions and appointments were provided for those considered likely to benefit from non-surgical management. Following assessment, patients were referred to an appropriate healthcare professional or were discharged. At the initial appointment or following a period of care, patients were discharged if non-surgical management was successful, surgery was not indicated, patients did not want surgery, and if patient's failed to attend their appointments. All other patients were referred for an orthopaedic consultation as indicated.

Results: Ninety-five of the 100 patients (69 females and 31 males; mean age 51.9, SD 16.4 years) attended their appointment at the podiatry-led assessment service. The 95 referrals contained a total of 107 diagnoses, of which the podiatrist agreed with the diagnosis stated on the referral in 56 cases (Kappa $=0.49$, SE $=0.05$ ). Overall, 34 of the 100 patients were referred to an orthopaedic surgeon and the remaining 66 patients were discharged from the orthopaedic waiting list without requiring an orthopaedic consultation.

Conclusions: Two-thirds of patients who had an appointment at the podiatry-led assessment service were discharged without requiring a surgical consultation. The introduction of a podiatry-led service assists with timely provision of patient care and ensures those with the greatest need for orthopaedic surgery have improved access to specialist care.

\section{Background}

Foot pain affects approximately one in five people in the general community and it is associated with reduced health-related quality of life [1] and self-reported disability [2]. Although many musculoskeletal foot conditions can be managed with non-surgical interventions [3,4], recalcitrant

\footnotetext{
* Correspondence: d.bonanno@latrobe.edu.au

'Department of Podiatry, Faculty of Health Sciences, La Trobe University, Bundoora, VIC 3086, Australia

${ }^{2}$ Lower Extremity and Gait Studies program, Faculty of Health Sciences, La Trobe University, Bundoora, VIC 3086, Australia

Full list of author information is available at the end of the article
}

and complex cases can require surgery [5]. In Australia, the demand for foot and ankle orthopaedic services in public health settings currently outweighs capacity due to factors such as limited theatre availability, competing surgical priorities and a limited surgical workforce [6]. With a growing and ageing population in Australia, the future demand for foot and ankle surgery is expected to increase [7].

Several initiatives have been proposed to help overcome the problem of unmet surgical demand in orthopaedic units [6], with one being the introduction of experienced allied health professionals to perform some roles traditionally provided by orthopaedic surgeons [8-11]. Allied health 
professionals in these positions require specialty area expertise, often have completed relevant post-graduate studies, and have the competency to maximise their scope of practice in a public outpatient setting [8-11]. The roles they perform can include triaging orthopaedic referrals, assessing patients, and establishing and initiating a management plan [8-11]. Introducing experienced allied health professionals into orthopaedic units can be relatively seamless to implement as it utilises the skills of an existing workforce [6]. The current evidence for using allied health professionals in these roles is generally promising with studies indicating high patient and referrer satisfaction [9,11-14], reductions in waiting time $[8,11]$, high agreement in diagnosis and management decisions with orthopaedic surgeons $[9,15]$, and a high proportion of patients being assessed, managed and discharged without requiring a surgical consultation $[8,9,11]$.

Although the use of allied health professionals in orthopaedic clinics is well documented, only two studies have investigated the role of a podiatrist working in this capacity in Australia; and they have demonstrated reductions in patient waiting times [8], improved service efficiency and high patient satisfaction [14]. The aim of this clinical audit was to evaluate the outcomes of introducing a podiatrist to triage, assess and initiate the management of patients referred to an orthopaedic outpatient unit with a foot or ankle condition. The primary outcome was to determine the proportion of patients that had an appointment at the podiatry-led assessment service to be discharged without requiring an orthopaedic appointment. Secondary outcomes include determining the: (i) agreement in diagnosis written on the patient referral compared with that of the assessing podiatrist, (ii) proportion of foot and ankle conditions presenting to the service, and (iii) proportion of each condition to require an orthopaedic appointment.

\section{Methods}

This study was a retrospective clinical audit of the first 100 patients to receive an appointment at a new podiatry-led assessment service in the orthopaedic outpatient department at The Northern Hospital, a public hospital located in an outer northern suburb of Melbourne. The podiatrist (DRB) responsible for triaging referrals and assessing patients has over 10 years of clinical experience, completed postgraduate studies (Postgraduate Diploma in Research Methodology) and has several peer-reviewed publications relating to the assessment and management of musculoskeletal conditions of the foot and lower limb.

Patients referred for a surgical consultation were initially triaged by orthopaedic surgeons into different categories of surgical priority (Table 1). Referrals were classified as 'Category 3 ' if the condition was considered unlikely to deteriorate quickly or had no potential to become an emergency. The podiatrist subsequently triaged all existing and new 'Category 3' referrals consisting of musculoskeletal foot and ankle conditions. Those considered likely to benefit from non-surgical management were provided with an appointment at the podiatry-led assessment service.

Patients were independently assessed by the podiatrist, with an orthopaedic surgeon generally being available for consultation if required. A standardised assessment form was used to record health-related information and details specific to the patient's condition(s) as per standard practice. Patients considered likely to benefit from non-surgical interventions were referred to an appropriate healthcare professional, as determined by the assessing podiatrist, and a management plan was established. Due to the variety of conditions expected to be seen, no standardised treatment protocols were utilised and all initial management decisions were based on the podiatrist's clinical reasoning in context of each patient and evidence based practice. Patients identified as requiring a surgical opinion were escalated for an appointment with an orthopaedic surgeon.

Patients were discharged from the orthopaedic waitlist with patient consent when: (i) non-surgical treatment was successful in resolving the presenting problem, (ii) if surgery was not indicated, either for the condition or due to comorbidities, and (iii) if patients indicated they did not want surgery. In addition, patients were discharged if they failed to attend their appointment on two or more occasions without prior communication. Patients that did not adequately respond to non-surgical interventions were referred for an orthopaedic consultation if clinically indicated.

\section{Data collection and analysis}

All data were obtained from the Northern Health electronic patient records and consisted of information recorded as per standard practice. Data collected included patient demographics, referrer and podiatrist diagnosis, and the patient's referral and treatment pathway. Inter-rater agreement between the diagnosis provided on the referral and the triaging podiatrist was determined using the Cohen's kappa statistic. All data were de-identified prior to analysis and were analysed using IBM SPSS (version 21.0). This clinical audit was considered a quality assurance activity that did not require ethical review in accordance with the National Statement on Conduct in Human Research (NHMRC 2007).

\section{Results}

\section{Profile of patients and conditions}

The 100 patients were provided an appointment at the podiatry-led assessment service between June 2012 and April 2013. The patients consisted of 69 females and 31 males, with a mean age of 51.9 (SD 16.4) years. Of the 100 patients, five failed to attend their appointment on two or more occasions and were discharged (Figure 1). Of the remaining 95 patients, their referrals contained a total of 107 diagnoses (some patients had more than one diagnosis), of 
Table 1 Hospital waiting list categories

\begin{tabular}{lll}
\hline Category & Description & $\begin{array}{l}\text { Recommended } \\
\text { admission time }\end{array}$ \\
\hline 1 & Patient has a condition that has potential to deteriorate rapidly to the point it may become an emergency & Within 30 days \\
2 & $\begin{array}{l}\text { Patient has a condition that is causing some pain, dysfunction or disability, but is unlikely to deteriorate quickly } \\
\text { or become an emergency }\end{array}$ & Within 90 days \\
3 & $\begin{array}{l}\text { Patient has a condition that is causing minimal or no pain, dysfunction or disability, which is also unlikely to } \\
\text { deteriorate quickly or has no potential to become an emergency }\end{array}$ & Within 365 days \\
\hline
\end{tabular}

which 56 were agreed upon by the triaging podiatrist (Kappa $=0.49, \mathrm{SE}=0.05)$. The podiatrist diagnosed 20 different conditions, with the most common diagnoses being hallux valgus $(n=23)$, plantar fasciitis $(n=21)$, intermetatarsal neuroma or bursitis $(n=13)$, tibialis posterior tendinopathy $(n=7)$, and Achilles tendinopathy $(n=5)$. A summary of all diagnoses $(n=104)$ made by the podiatrist is provided in Table 2 .

\section{Patient destination}

Following the assessment of the 95 patients at the podiatryled assessment service, 6 were discharged without receiving further treatment or requiring further referral(s). Of the remaining 89 patients, a total of 102 referrals were made following the initial appointment. Referrals were sent to a podiatrist $(n=72)$, orthopaedic surgeon $(n=18)$, orthotist $(\mathrm{n}=5)$, physiotherapist $(\mathrm{n}=3)$, diabetes educator $(\mathrm{n}=1)$, occupational therapist $(n=1)$, gerontologist $(n=1)$ and rheumatologist $(n=1)$. After a period of receiving nonsurgical care, a further 16 patients were referred to an orthopaedic surgeon. Overall, a total of 34 of the 100 patients were referred to an orthopaedic surgeon and the remaining 66 patients were discharged from the orthopaedic waiting list without requiring an orthopaedic consultation (Figure 1).

\section{Surgical referral for various conditions}

The 34 patients referred for a surgical opinion were diagnosed by the podiatrist with 17 different conditions. Of interest, 15 of these patients had a diagnosis stated on their initial referral that was not agreed upon by the podiatrist. The most common diagnoses provided by the podiatrist that was referred for a surgical opinion were hallux valgus $(n=9)$, inter-metatarsal neuroma or bursitis $(n=5)$, plantar fasciitis $(n=3)$, hallux rigidus $(n=3)$, a variety of arthropathies $(n=3)$ and plantar plate tears $(n=2)$ (Table 2).

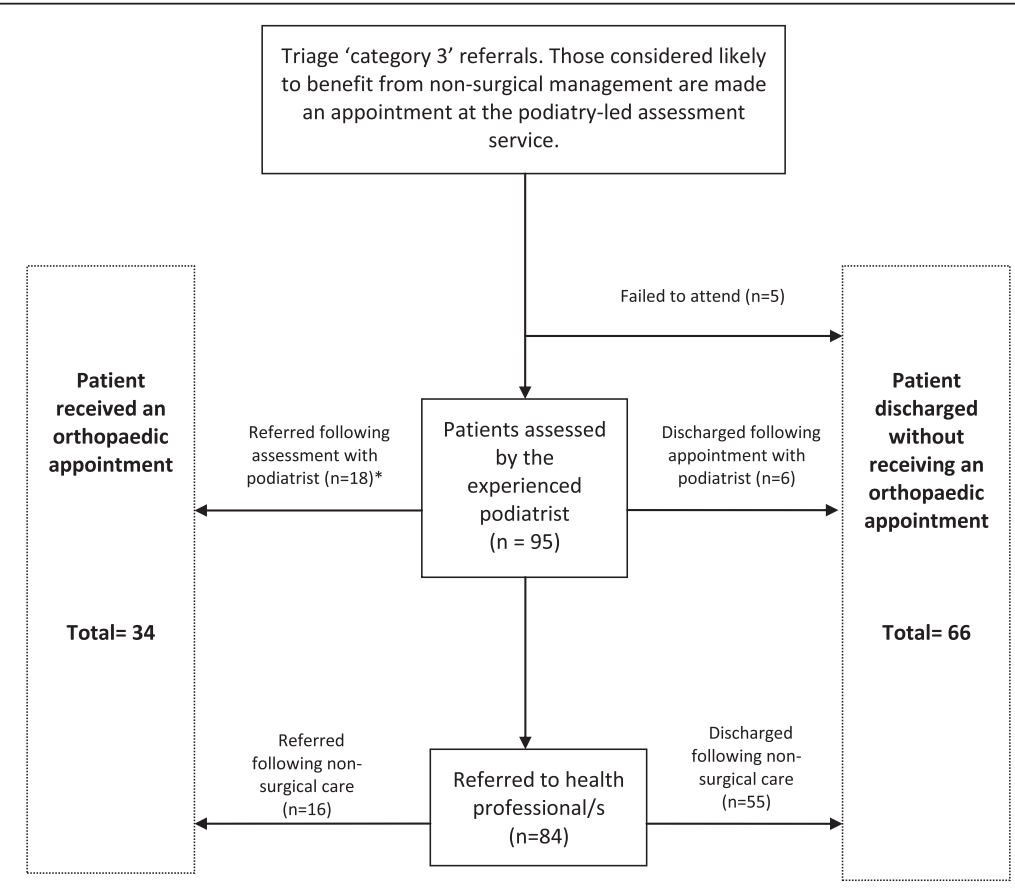

*Some patients were referred to an orthopaedic surgeon and other health professional/s following their assessment with the podiatrist.

Figure 1 Flowchart of patients attending the podiatry-led assessment service. 
Table 2 Diagnoses $(n=104)$ of patients $(n=95)$ as determined by the podiatrist and the number of each diagnosis to require an orthopaedic appointment

\begin{tabular}{|c|c|c|c|}
\hline Condition & $\begin{array}{l}\text { Number of presentations } \\
\mathrm{n} \text { (\% of total diagnoses) }\end{array}$ & $\begin{array}{l}\text { Required orthopaedic appointment } \\
\text { n (\% per condition) }\end{array}$ & $\begin{array}{l}\text { Did not require orthopaedic appointment } \\
\mathrm{n} \text { (\% per condition) }\end{array}$ \\
\hline Hallux valgus & $23(22.1 \%)$ & $9(39.1 \%)$ & $14(60.9 \%)$ \\
\hline Plantar fasciitis & $21(20.1 \%)$ & $3(14.3 \%)$ & $18(85.7 \%)$ \\
\hline Inter-metatarsal neuroma/bursitis & $13(12.5 \%)$ & $5(38.5 \%)$ & $8(61.5 \%)$ \\
\hline Tibialis posterior tendinopathy & $7(6.7 \%)$ & $1(14.3 \%)$ & $6(85.7 \%)$ \\
\hline Achilles tendinopathy & $5(4.8 \%)$ & $1(20.0 \%)$ & $4(80 \%)$ \\
\hline Plantar plate tear & $4(3.8 \%)$ & $2(50.0 \%)$ & $2(50 \%)$ \\
\hline $\begin{array}{l}\text { Neuralgic pain (origin extrinsic to } \\
\text { foot) }\end{array}$ & $4(3.8 \%)$ & $1(25.0 \%)$ & $3(75 \%)$ \\
\hline Hallux rigidus & $3(2.9 \%)$ & $3(100 \%)$ & $0(0 \%)$ \\
\hline Midfoot osteoarthritis & $3(2.9 \%)$ & $1(33.3 \%)$ & $2(66.6 \%)$ \\
\hline Digital deformity & $3(2.9 \%)$ & $1(33.3 \%)$ & $2(66.6 \%)$ \\
\hline Degenerative arthropathy & $3(2.9 \%)$ & $3(100 \%)$ & $0(0 \%)$ \\
\hline Peroneal tendinopathy & $3(2.9 \%)$ & $1(33.3 \%)$ & $2(66.6 \%)$ \\
\hline Synovitis/capsulitis & $2(1.9 \%)$ & $1(50.0 \%)$ & $1(50 \%)$ \\
\hline Inflammatory arthritis & $2(1.9 \%)$ & $1(50.0 \%)$ & $1(50 \%)$ \\
\hline Dermatological & $2(1.9 \%)$ & $0(0 \%)$ & $2(100 \%)$ \\
\hline Sesamoiditis & $1(1.0 \%)$ & $0(0 \%)$ & $1(100 \%)$ \\
\hline Ganglion & $1(1.0 \%)$ & $1(100.0 \%)$ & $0(0 \%)$ \\
\hline Retro-calcaneal bursitis & $1(1.0 \%)$ & $1(100.0 \%)$ & $0(0 \%)$ \\
\hline Sub-ungual exostosis & $1(1.0 \%)$ & $1(100.0 \%)$ & $0(0 \%)$ \\
\hline Extensor tendinopathy & $1(1.0 \%)$ & $0(0 \%)$ & $1(100 \%)$ \\
\hline Diagnosis not determined & $1(1.0 \%)$ & $0(0 \%)$ & $1(100 \%)$ \\
\hline Total & $104(100 \%)$ & $36(34.6 \%)$ & $68(65.4 \%)$ \\
\hline
\end{tabular}

\section{Discussion}

This clinical audit evaluated the effects of introducing an experienced podiatrist to initiate the triage, assessment and management of patients referred to an orthopaedic outpatient unit with a foot or ankle condition. The findings of this study indicate that two-thirds of the patients who had an appointment at the podiatry-led assessment service were removed from the orthopaedic waitlist without requiring a surgical consultation. These findings are similar, albeit greater, to previous studies involving podiatrists performing assessment and triage roles where $41-45 \%$ of patients were discharged without requiring surgical management $[8,14]$. Current literature suggests allied health professionals, including podiatrists, can ease demands placed on orthopaedic outpatient services $[8,9,14,16]$. Importantly, as 'Category 3' patients generally wait the longest for a surgical consultation, the assessment and triage service allows those considered likely to benefit from nonsurgical interventions to be redirected, in many cases earlier, for effective treatment.
The triage of referrals is intended to ensure patients receive appropriate and timely care, yet this process is highly reliant on referrals being accurate and informative. The results from this study indicate that almost half of the referrals received provided a non-specific diagnosis or one that wasn't agreed upon by the triaging podiatrist. Although many of the diagnostic disagreements between the referrer and the assessing podiatrist are likely to be true differences in opinion, there is the possibility that in some cases the condition may have changed in the time between the date of the referral and initial appointment. When disagreement is present, it often remains uncertain which diagnosis is most accurate as a definitive diagnosis is not always established. With this in mind, it should be noted that the triaging podiatrist was more likely to provide a more specific diagnosis than that contained on the referrals. Although all patients provided an appointment at the podiatry-led assessment service were considered likely to benefit from non-surgical intervention based on their referral, approximately $20 \%$ were directly referred to a surgeon following their initial 
assessment. In addition, nearly half of all patient referrals to receive an appointment with a surgeon were found to have a non-specific diagnosis or one that wasn't agreed upon by the podiatrist. This demonstrates the propensity for a referral lacking detail and/or accuracy to delay appropriate surgical treatment when indicated. Based on our findings, we recommend referrers ensure they provide informative and specific referrals to maximise triage efficiency and accuracy.

A novel aspect of the present study is it reported the specific conditions seen at the podiatry-led assessment service and the proportion of these conditions that were discharged with and without requiring a surgical opinion. This information can potentially be used in several ways. First, referrers could be advised that specific foot conditions, such as plantar fasciitis, should be referred for non-surgical management as a first-line of care as supported by this study and, more importantly, high quality clinical trials and clinical practice guidelines $[4,17,18]$. This would allow patients to receive appropriate, timely care and reduce unnecessary referrals to orthopaedic services, with the latter further reducing demand on surgical services. In contrast, for conditions such as hallux rigidus, it would be reasonable for referrers to strongly consider a surgical referral if the condition remains recalcitrant following non-surgical management $[5,19]$. Second, when all new referrals are being triaged, conditions likely to benefit from non-surgical and surgical interventions could be identified and the most appropriate treatment options and referrals could be established. Importantly, this information could be applied at various stages of triage including the initial entry points into a public health service. If these changes were implemented it could potentially improve the efficiency of how foot and ankle referrals are triaged and managed.

Although the preliminary findings of this audit are promising, further service efficiencies are likely and should be considered. As triage prioritisation systems have been shown to have issues with reliability [20,21], podiatrists could potentially triage all referrals with foot and ankle conditions to assist with identifying prioritisation categories and provide patients with early intervention where possible. By reducing the demands of triage on orthopaedic surgeons, it could potentially allow surgeons more time to consult patients already triaged and identified as requiring their surgical skills.

The findings of this audit should be considered in light of several limitations. First, and most importantly, the lack of a comparison group introduces uncertainty around whether the results reported are the effect of the new podiatry-led assessment service or unrelated factors. In addition, as assessment and patient care was not controlled, the results of this audit may be influenced by the individual clinicians rather than the new initiative itself. Nevertheless, in light of the limitations, the findings of this audit can be viewed as additional evidence to support the introduction of podiatry-led assessment services in orthopaedic units. In addition to addressing the aforementioned limitations, future research should consider measuring outcomes such as cost-benefit, waiting times, patient and referrer satisfaction and validated measures of changes in pain, function and psychological well-being.

\section{Conclusion}

The majority of patients referred to an orthopaedic outpatient unit with a foot or ankle condition who were provided an appointment at the podiatry-led assessment service were discharged without requiring a surgical appointment. The introduction of the podiatry-led assessment service may assist with improving the efficiency of care, which is provided by the most appropriate health professional, to patients referred for a surgical consultation with a foot and ankle condition.

\section{Competing interests}

The authors declare that they have no competing interests.

\section{Authors' contributions}

All authors were fully involved in the preparation of the study procedures. $J G, A A S, D R B$ and VGB conceived the study and participated in the design and coordination of this study. DRB, VGB and DST were involved in data collection and data analysis. DRB and DST were responsible for the preparation of the manuscript with all other authors involved in its review prior to submission for publication. The material within has not been and will not be submitted for publication elsewhere. All authors read and approved the final manuscript.

\section{Author details}

${ }^{1}$ Department of Podiatry, Faculty of Health Sciences, La Trobe University, Bundoora, VIC 3086, Australia. 'Lower Extremity and Gait Studies program, Faculty of Health Sciences, La Trobe University, Bundoora, VIC 3086, Australia. ${ }^{3}$ Podiatry Department, The Northern Hospital, Epping, VIC 3076, Australia.

Received: 25 June 2014 Accepted: 20 October 2014

Published online: 18 November 2014

\section{References}

1. Hill CL, Gill TK, Menz HB, Taylor AW: Prevalence and correlates of foot pain in a population-based study: the North West Adelaide health study. J Foot Ankle Res 2008, 1(2):1-7.

2. Keysor JJ, Dunn JE, Link CL, Badlissi F, Felson DT: Are foot disorders associated with functional limitation and disability among community-dwelling older adults? J Aging Health 2005, 17(6):734-752.

3. Kulig K, Reischl SF, Pomrantz AB, Burnfield JM, Mais-Requejo S, Thordarson $D B$, Smith RW: Nonsurgical management of posterior tibial tendon dysfunction with orthoses and resistive exercise: a randomized controlled trial. Phys Ther 2009, 89(1):26-37.

4. Landorf KB, Keenan AM, Herbert RD: Effectiveness of foot orthoses to treat plantar fasciitis: a randomized trial. Arch Intern Med 2006, 166(12):1305-1310.

5. Torkki M, Malmivaara A, Seitsalo S, Hoikka V, Laippala P, Paavolainen P: Surgery vs orthosis vs watchful waiting for hallux valgus: a randomized controlled trial. JAMA 2001, 285(19):2474-2480.

6. Exploring solutions to better meet demand for foot and ankle surgical services: report for the Department of Human Services. The Australian Health Workforce Institute. Victoria; 2009.

7. Menz HB, Gilheany MF, Landorf KB: Foot and ankle surgery in Australia: a descriptive analysis of the medicare benefits schedule database, 1997-2006. J Foot Ankle Res 2008, 1(1):1-10.

8. Homeming $L$, Kuipers $P$, Nihal A: Orthopaedic podiatry triage: process outcomes of a skill mix initiative. Aust Health Rev 2012, 36(4):457-460.

9. Oldmeadow LB, Bedi HS, Burch HT, Smith JS, Leahy ES, Goldwasser M: Experienced physiotherapists as gatekeepers to hospital orthopaedic outpatient care. Med J Aust 2007, 186(12):625-628. 
10. Harding KE, Taylor NF, Leggat SG, Stafford M: Effect of triage on waiting time for community rehabilitation: a prospective cohort study. Arch Phys Med Rehabil 2012, 93(3):441-445.

11. Samsson K, Larsson MEH: Physiotherapy screening of patients referred for orthopaedic consultation in primary healthcare - a randomised controlled trial. Man Ther 2014, 19(5):386-391.

12. Kennedy DM, Robarts S, Woodhouse L: Patients are satisfied with advanced practice physiotherapists in a role traditionally performed by orthopaedic surgeons. Physiother Can 2010, 62(4):298-305.

13. Bath B, Janzen B: Patient and referring health care provider satisfaction with a physiotherapy spinal triage assessment service. J Multidiscip Healthc 2012, 5:1-15.

14. Walsh TP, Pilkington DC, Wong EJ, Brown CH, Mercer GE: Orthopaedic triaging by podiatrists: a prospective study of patient satisfaction and service efficiency. Aust Health Rev 2014, 38(4):406-411.

15. Gardiner J, Turner P: Accuracy of clinical diagnosis of internal derangement of the knee by extended scope physiotherapists and orthopaedic doctors: retrospective audit. Physiother 2002, 88(3):153-157.

16. Blackburn MS, Nall C, Cary B, Cowan SM: Physiotherapy-led triage clinic for low back pain. Aust Health Rev 2009, 33(4):663-670.

17. McPoil TG, Martin RL, Cornwall MW, Wukich DK, Irrgang JJ, Godges JJ: Heel pain-plantar fasciitis: clinical practice guildelines linked to the international classification of function, disability, and health from the orthopaedic section of the American physical therapy association. J Orthop Sports Phys Ther 2008, 38(4):A1-A18.

18. McMillan AM, Landorf KB, Gilheany MF, Bird AR, Morrow AD, Menz HB: Ultrasound guided corticosteroid injection for plantar fasciitis: randomised controlled trial. BMJ 2012, 344:e3260.

19. Vanore JV, Christensen JC, Kravitz SR, Schuberth JM, Thomas JL, Weil LS, Zlotoff HJ, Mendicino RW, Couture SD: Diagnosis and treatment of first metatarsophalangeal joint disorders. section 1: hallux valgus. J Foot Ankle Surg 2003, 42(3):112-123.

20. Harding KE, Taylor NF, Leggat SG, Wise VL: Prioritizing patients for community rehabilitation services: do clinicians agree on triage decisions? Clin Rehabil 2010, 24(10):928-934.

21. Harding KE, Taylor NF, Leggat SG, Wise VL: A training programme did not increase agreement between allied health clinicians prioritizing patients for community rehabilitation. Clin Rehabil 2011, 25(7):599-606.

doi:10.1186/s13047-014-0045-6

Cite this article as: Bonanno et al:: Evaluating the outcomes of a podiatry-led assessment service in a public hospital orthopaedic unit. Journal of Foot and Ankle Research 2014 7:45.

\section{Submit your next manuscript to BioMed Central and take full advantage of:}

- Convenient online submission

- Thorough peer review

- No space constraints or color figure charges

- Immediate publication on acceptance

- Inclusion in PubMed, CAS, Scopus and Google Scholar

- Research which is freely available for redistribution 